\title{
Toracotomia Resucitativa Por Trauma Cardiaco Penetrante En Recien Nacido Reporte De Un Caso Excepcional
}

\author{
Napoleón Méndez ${ }^{1}$, Guillermo López ${ }^{2}$
}

\section{RESUMEN}

Antecedentes: La toracotomía resucitativa es una maniobra realizada en los pacientes con traumatismos que se presentan "in extremis," especialmente en lesiones penetrantes del torso. Existen numerosos reportes en literatura sobre toracotomía en adultos, algunos en pediatría, pero no hay reportes de casos en recién nacidos debido a lo inusual del trauma en esta etapa de la vida.

Objetivo: El presente reporte tiene como objetivo documentar un caso inusual de un recién nacido con traumatismo penetrante por bala en el tórax, nacido por una cesárea de urgencia debido al estado agonal de su madre por las múltiples lesiones que sufrió. Al encontrarse en estado fatal se intenta resucitarlo por medio de una toracotomía anterolateral izquierda, no se logra obtener la sobrevida debido a lo irreparable de la lesión y las consecuencias de la exanguinación.

Resultados: El presente caso documenta el ingreso al servicio de Emergencia de adultos de una paciente embarazada, clínicamente a término con múltiples perforaciones por arma de fuego en tórax y abdomen, en estado agónal debido a las pérdidas hemáticas masivas. Se logra auscultar un adecuado foco fetal, por lo que el equipo de emergencia decide llevar a cesárea de emergencia, obteniendo un producto sin signos de vida al momento de extraerlo del útero (estado fatal). Se realiza toracotomía de resucitación en el recién nacido evidenciando una lesión irreparable del ventrículo izquierdo y hemorragia exanguinante.

Conclusiones: La toracotomía resucitativa en trauma tiene estrictas indicaciones y los mejores resultados se obtienen en el paciente con lesiones penetrantes en tórax, con paro presencial o de reciente evolución. Este caso evidencia una clara indicación del procedimiento: estado fatal, paro cardiaco potencialmente rescatable, traumatismo penetrante en el tórax; es poco frecuente en pediatría y no hay reportes de la literatura en recién nacidos. La decisión de realizar toracotomía no es discutible si la indicación es clara como el presente caso, y la edad no es un factor a considerar.

Palabras clave: Toracotomía de reanimación, trauma cardiaco, reporte de un caso, toracotomía de emergencia, lesiones por arma de fuego, herida precordial, trauma pediátrico, lesión cardiaca penetrante.

Keywords: Herida precordial, Heridas penetrantes, Lesión cardiaca penetrante, Lesión por arma de fuego, Reporte de un caso, Toracotomía de emergencia, Toracotomía de reanimación, Trauma cardiaco, Trauma pediátrico.

Panamerican Journal of Trauma, Critical Care \& Emergency Surgery (2021): 10.5005/jp-journals-10030-1354

\section{Antecedentes}

En trauma la gravedad de las lesiones y la pérdida de sangre pueden llevar al individuo a un paro cardiorespiratorio de desarrollo inmediato, debido a la rapidez de los violentos cambios de la fisiología debido a la intensidad de las repercusiones de las lesiones. En trauma grave un alto porcentaje de las víctimas fallecen en la escena del trauma o en el trasporte, y los que logran sobrevivir llegan en estado moribundo o grave. Una de las maniobras que puede ser salvar la vida del paciente gravemente traumatizado es la toracotomía. La toracotomía es un procedimiento descrito desde hace más de 50 años en el paciente moribundo por trauma como maniobra para salvar la vida., ${ }^{1,2} \mathrm{El}$ procedimiento debe ser selectivo para mejorar los resultados de sobrevida, ${ }^{3}$ en sus inicios la toracotomía resucitativa se realizaba liberalmente, incluso en el paro cardiorespiratorio de origen médico; con el tiempo se fue evidenciando que la sobrevida dependía de algunas variables, y el realizarlo liberalmente no conseguía buenos resultados y no era costo efectivo. Existe mejor sobrevida en los casos de trauma penetrante comparados con los traumatismos cerrados, $(2,4-6)$ no existe contraindicación absoluta si el escenario es un traumatismo cerrado con paro presencial o reciente; pero se sabe que la sobrevida no supera el $1 \%$, contrario a los traumatismos penetrantes que tienen mejores resultados. Los pacientes que se practica toracotomía por lesiones en la región torácica tienen
${ }^{1-2}$ CirugíA De Trauma, Hospital General San Juan De Dios, Guatemala, Guatemala, Guatemala

Corresponding Author: Napoleón Méndez, CirugíA De Trauma, Hospital General San Juan De Dios, Guatemala, Guatemala, Guatemala, Phone: +50190620, e-mail: napoleonmendez1971@gmail.com

How to cite this article: Méndez N, López G. Toracotomia Resucitativa Por Trauma Cardiaco Penetrante En Recien Nacido Reporte De Un Caso Excepcional. Panam JTrauma Crit Care Emerg Surg 2021;10(3):130-133. Source of support: Nil

Conflict of interest: None

mejor pronóstico que los que tienen trauma extratorácico, sobre todo si tienen lesión de corazón. ${ }^{2,7,8}$ La presencia de signos de vida ${ }^{2,8,9}$ y adecuada resucitación cardiopulmonar en el traslado ${ }^{9,10}$ también son factores pronósticos y se han convertido en indicadores de realizar la toracotomía. La presencia de signos de vida mejora el pronóstico, y si el paciente tiene un paro presencial reciente puede considerarse un paro potencialmente salvable; sobre todo si el paciente recibe maniobras adecuadas de resucitación y no rebasa los 15 minutos en trauma penetrante. Mientras más tiempo de paro el pronóstico es peor. Es importante seguir las indicaciones y no abusar del procedimiento en pro de los buenos resultados del paciente y los costos. ${ }^{11}$ sin embargo, si el

() The Author(s). 2021 Open Access This article is distributed under the terms of the Creative Commons Attribution 4.0 International License (https://creativecommons. org/licenses/by-nc/4.0/), which permits unrestricted use, distribution, and non-commercial reproduction in any medium, provided you give appropriate credit to the original author(s) and the source, provide a link to the Creative Commons license, and indicate if changes were made. The Creative Commons Public Domain Dedication waiver (http://creativecommons.org/publicdomain/zero/1.0/) applies to the data made available in this article, unless otherwise stated. 
paciente cumple las indicaciones no debe vacilarse en realizar de inmediato el procedimiento y darle la oportunidad de sobrevida (Figs 1 and 2).

Debe ser un procedimiento metódico y deben de seguirse todos los pasos, desde el adecuado manejo de la vía aérea, la incisión que se realiza que para resucitación es indicada la anterolateral izquierda por su exposición, facilidad de entrada y accesibilidad que consigue. La pericardiotomía es el siguiente paso y tiene como objetivo aliviar el taponamiento que el paciente pueda tener. El control de hemorragia cardíaca se puede conseguir desde el control digital en la herida hasta uso de clamps o suturas en los defectos. Para las lesiones sangrantes extra-cardíacas especialmente extratorácicas por debajo del diafragma el clampeo de la aorta torácica descendente la técnica recomendada. ${ }^{3,12}$ La sobrevida del procedimiento de toracotomía varía entre 7 y 14\%. 3,12-15

El trauma pediátrico es frecuente, y los escenarios de lesiones severas pueden ser similares que en los adultos. Las lesiones cerradas devastadoras y el trauma penetrante es menos frecuente en los niños que en los adultos, pero hay una frecuencia importante y puede ser tan demandante como en el adulto. En la población pediátrica la toracotomía resucitativa es un procedimiento utilizado y ampliamente documentado. ${ }^{16,17}$ Si bien es cierto en el manejo del trauma pediátrico el manejo conservador se debe preferir en los casos que sea posible, las lesiones graves deben de tratarse con la inmediatez y agresividad que se tratan en el adulto. Los pacientes que por su gravedad desarrollan un choque profundo, resultan agónicos o deterioran a paro cardiorespiratorio deben de practicarse maniobras salvadoras como la toracotomia resucitativa. La sobrevida es variable y parecida a la población adulta entre 0 y $26 \% .{ }^{16,18,8}$ Los pacientes con trauma penetrante graves que necesitan toracotomía también mejores resultados en la población pediátrica que los que tienen trauma cerrado, ya que el trauma cerrado tiene pobres resultados en ambos grupos. Sin embargo, el mecanismo es menos frecuente en esta población y esto puede influir en los resultados generales de sobrevida. ${ }^{16,20} \mathrm{En}$ la edad neonatal no se describen casos de toracotomía por trauma. Existen reportes de casos en edades muy tempranas, pero no en recién nacidos.

\section{Metodologia}

El objetivo del presente estudio es hacer una descripción de caso clínico, por lo excepcional del mismo. Se hace la descripción basada

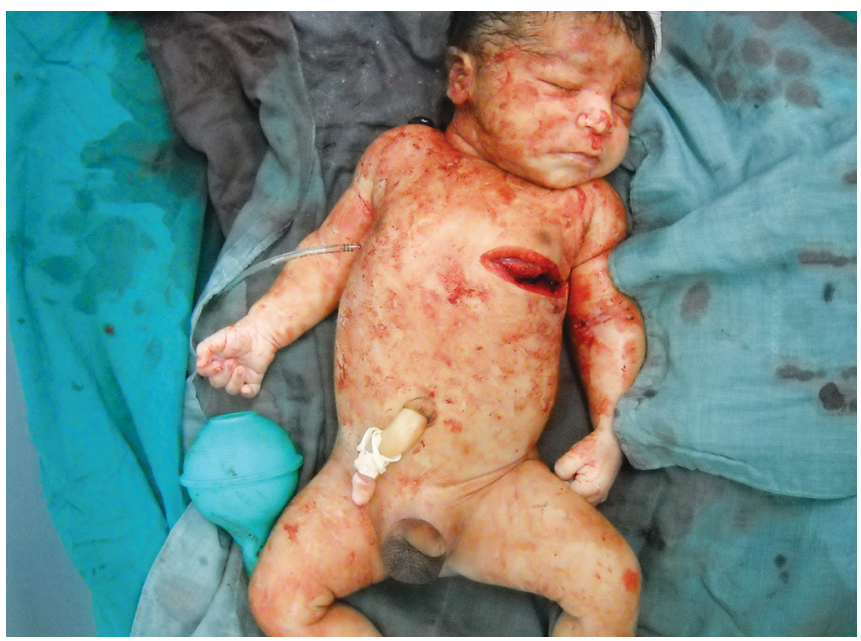

Fig 1: fotografía de incisión de toracotomiía realizada en la observación y atención directa del caso por los autores del artículo, además de la documentación en el historial clínico.

\section{Resultados}

\section{Descripcion Del Caso}

Se recibe en la emergencia de adultos una paciente mujer de aproximadamente 25 años quien presenta múltiples lesiones por proyectil por arma de fuego, con orificios de entrada y salida de heridas en el torso. Debido a la gravedad no se pudieron obtener datos de antecedentes del trauma o antecedentes médicos de la paciente.

La víctima de trauma en estado agónico sin presión arterial audible, pulsos periféricos no palpables, pálida, sudorosa, no responde a estímulos verbales. se ausculta foco cardíaco. Responde a estímulos dolorosos con movimientos de localización al dolor.

Abdomen con útero grávido por arriba del ombligo pronunciándose casi al epigastrio clínicamente a término.

- Se ausculta foco cardíaco fetal en 160 x ' distinto al foco central (120x')

- Paciente tiene esfuerzo respiratorio y responde al dolor con movimientos

- Se realiza la atención $A B C D E$ y se prepara para realizar cesárea de urgencia, se avisa a ginecología.

- Se inicia la resucitación materna durante inducción de choque, y protocolo de transfusión.

Se realiza cesárea transperitoneal por equipo de cirugía, ya que por la premura ginecólogos no logran llegar a tiempo al proceso quirúrgico. Se aborda a través de línea media laparotomía amplia: se documenta abundante hemoperitoneo y 3 penetraciones al útero que está activamente sangrando. Se realiza abordaje del útero con insición corpórea se obtiene producto recién nacido masculino a término con apgar $0 / 0$ con herida por arma de fuego en tórax izquierdo. El bebé tenia foco cardiaco fetal documentado al recibir a la madre en urgencias y al nacimiento sin signos de vida cumple el criterio de estado fatal por lo que se realiza intubación orotraqueal. Debido a ser un trauma penetrante en tórax, con paro reciente, estado fatal y posibilidades de vida se realiza toracotomía intubación orotraqueal y posterior toracotomía resucitativa a través de incisión anterolateral izquierda, se documenta lesión destructiva

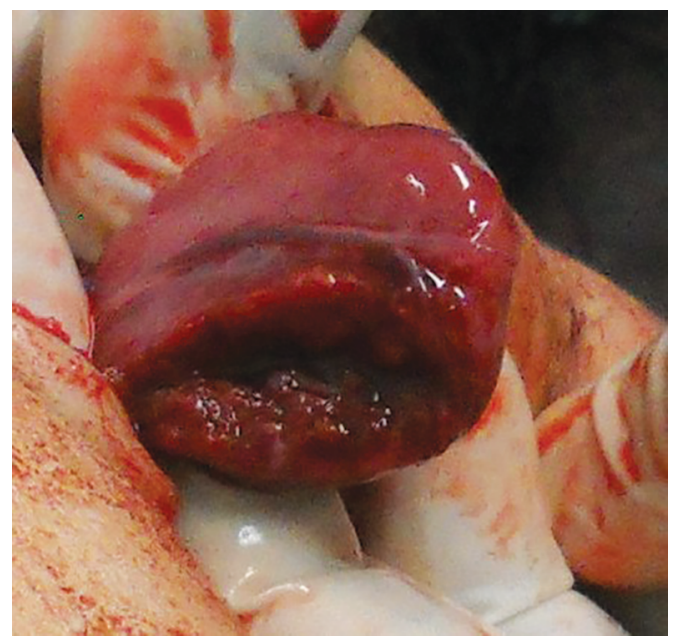

Fig 2: fotografía de la lesión cardiaca 
irreparable del ventrículo izquierdo con hemorragia exanguinante, por lo que se declara la muerte del recién nacido. Posteriormente se atiende a la madre quien se documenta hemorragía exanguinante, lesiones de múltiples órganos entre los cuales tiene lesión de grandes vasos abdominales; se realiza también toracotomía resucitativa sin resultados positivos.

\section{Discussion}

La toracotomía resucitativa es un procedimiento que puede salvar vidas, puede ser la ultima oportunidad que tenga una víctima de trauma grave. Existen amplios reportes del beneficio y la sobrevida del procedimiento, que aunque no son altos puede tener mejores resultados si se siguen las indicaciones y se realiza con los pasos recomendados y cumpliendo los objetivos descritos en la técnica. En la población pediátrica se cumplen las mismas indicaciones y la sobrevida es parecida. A pesar de ser un procedimiento agresivo da la oportunidad al niño lesionado a tener una posibilidad, aunque sea mínima la sobrevida.

A pesar de la controversia en adultos como en pediatría en la que algunos condenan la toracotomía, existen indicaciones claras de la misma que son recomendadas incluso en guías de manejo. Al revisar la literatura existe amplia documentación de la toracotomía en la población pediátrica, incluso en edades muy tempranas, debido a que la población pediátrica no esta exenta de ser victima de lesiones traumáticas graves que requieren dicho procedimiento. Sin embargo, en recién nacidos no está descrita esta necesidad. El trauma penetrante es menos frecuente en pediatría, por lo tanto, la toracotomía resucitativa es menos frecuente y tiene resultados influenciados por esta epidemiología. Por las características epidemiológicas y sociales el trauma neonatal es impensable y por lo tanto no se encuentran datos de mortalidad neonatal en niños recién nacidos que hayan necesitado toracotomía después del nacimiento por cesárea de urgencia. En una revisión publicada del banco de datos de Estados Unidos se documentaron 5,463 muertes pediátricas por trauma en un período del 2007 al 14. 4,549 casos por trauma cerrado, $83.27 \%$. Se documentaron 633 toracotomías (11.89\%) pero ningún caso en edad neonatal. ${ }^{22} \mathrm{Al}$ hacer una búsqueda en pub-med utilizando las palabras clave toracotomía resucitativa neonatal se obtienen 43 resultados, toracotomía neonatal por causas médicas en un caso anecdótico, ${ }^{23}$ pero ninguno por trauma penetrante. En la conducta de cesárea peri-morten fetal existen algunas documentaciones que favorecen el procedimiento si se compara con sola resucitación materna, sobre todo cuando más cercano de las 40 semanas tiene el producto. Sin embargo, en trauma penetrante no está descrito un caso documentado, y menos que haya necesitado toracotomía. Esto hace difícil la toma de decisiones en casos anecdóticos e impensables como el presentado en este artículo, y deben combinarse la evidencia clínica, el criterio médico, la experiencia y el sentido común. Es importante que estos casos tan infrecuentes y anecdóticos sean documentados para empezar a hacer la mejor evidencia y recomendaciones para futuras situaciones similares.

\section{Conclusion}

La toracotomía resucitativa en los pacientes con trauma es un procedimiento que puede salvar vidas, sobre todo si se siguen las indicaciones, se realiza en el paciente indicado y siguiendo un protocolo metódico. Los mejores resultados se obtienen en el traumatismo penetrante, el paro cardio-respiratorio presencial o reciente, la lesión torácica. En pediatría puede ser necesario recurrir a este procedimiento por las mismas indicaciones que la población adulta. En el feto que sufre lesión intra-útero en una edad con posibilidades de sobrevida deben de seguirse las mismas indicaciones de toracotomía que las descritas en otras edades, ya que las posibilidades de sobrevida a quien se le práctique justifica dicho procedimiento. este caso representa quizás uno de los primeros de toracotomía resucitativa por trauma en edad neonatal, y puede servir de guía para futuros casos.

\section{References}

1. Beall AC Jr, Diethrich EB, Cooley DA, et al. Surgical management of penetrating cardiovascular trauma. South Med J 1967;60:698-704. DOI: 10.1097/00007611-196707000-00005

2. Mattox KL, Espada R, Beall AC Jr, et al. Performing thoracotomy in the emergency center. JACEP 1974;3:13-17.

3. Seamon MJ, Haut ER, Van Arendonk K, et al. An evidence-based approach to patient selection for emergency department thoracotomy: a practice management guideline from the Eastern Association for the Surgery of Trauma. J Trauma Acute Care Surg 2015;79(1):159-73. DOI: 10.1097/TA.0000000000000648

4. Brown SE, Gomez GA, Jacobson LE, et al. Penetrating chest trauma: should indications for emergency room thoracotomy be limited? Am Surg 1996;62:530-533.

5. Karmy-Jones R, Nathens A, Jurkovich GJ, et al. Urgent and emergent thoracotomy for penetrating chest trauma. J Trauma 2004;56: 664-668. DOI: 10.1097/01.ta.0000068238.74552.4b

6. Sheppard FR, Cothren CC, Moore EE, et al. Emergency department resuscitative thoracotomy for nontorso injuries. Surgery 2006;139(4):574-576. DOI: 10.1016/j.surg.2005.12.005

7. Capote A, Michael A, Almodovar J, et al. Emergency department thoracotomy: too little, too much, or too late. Am SurgBr J Surg 2013;79:982-986. DOI: https://doi.org/10.1177/000313481307901005

8. Easter JS, Vinton DT, Haukoos JS. Emergent pediatric thoracotomy following traumatic arrest. Resuscitation 2012;83:1521-1524. DOI: 10.1016/j.resuscitation.2012.05.024

9. Lustenberger T, Labler L, Stover JF, et al. Resuscitative emergency thoracotomy in a Swiss trauma centre. Br J Surg 2012;99:541-548. DOI: 10.1002/bjs.7706

10. Johannesdottir BK, Mogensen B, Gudbjartsson T. Emergency thoracotomy as a rescue treatment for trauma patients in Iceland. Injury 2013;44(9):1186-1190. DOI: 10.1016/j.injury.2012.05.005

11. Passos EM, Engels PT, Doyle JD, et al. Societal costs of inappropriate emergency department thoracotomy. J Am Coll Surg 2012;214:18-25. DOI: 10.1016/j.jamcollsurg.2011.09.020

12. Burlew CC, Moore EE, Moore FA, et al. Western Trauma Association critical decisions in trauma: resuscitative thoracotomy. J Trauma Acute Care Surg 2012;73(6):1359-1363. DOI: 10.1097/TA.0b013e318270d2df. PMID: 23188227.

13. Boyd M, Vanek VW, Bourguet CC. Emergency room resuscitative thoracotomy: when is it indicated? J Trauma 1992;33(5):714-721. PMID: 1464921.

14. Branney SW, Moore EE, Feldhaus KM, et al. Critical analysis of two decades of experience with postinjury emergency department thoracotomy in a regional trauma center. J Trauma 1998;45(1):87-94; discussion 94-5. DOI: 10.1097/00005373-199807000-00019. PMID: 9680018.

15. Rhee PM, Acosta J, Bridgeman A, et al. Survival after emergency department thoracotomy: review of published data from the past 25 years. J Am Coll Surg 2000;190(3):288-298. DOI: 10.1016/s10727515(99)00233-1

16. Moore HB, Moore EE, Bensard DD. Pediatric emergency department thoracotomy: a 40-year review. J Pediatr Surg 2016;51(2):315-318. DOI: 10.1016/j.jpedsurg.2015.10.040. Epub 2015. PMID: 26597392 PMCID: PMC4960823. 
17. Allen $C J$, Valle EJ, Thorson $C M$, et al. Pediatric emergency department thoracotomy: a large case series and systematic review. J Pediatr Surg 2015;50:177-181. DOI: 10.1016/j.jpedsurg.2014.10.042

18. Boatright DH, Byyny RL, Hopkins E, et al. Validation of rules to predict emergent surgical intervention in pediatric trauma patients. J Am Coll Surg 2013;216:1094-1102.

19. Nicolson NG, Schwulst S, Esposito TA, et al. Resuscitative thoracotomy for pediatric trauma in Illinois, 1999 to 2009. Am J Surg 2015;210(4):720-723. DOI: 10.1016/j.amjsurg.2015.05.007. Epub 2015. PMID: 26206602.

20. Grisoni E, Stallion A, Nance ML, et al. The New Injury Severity Score and the evaluation of pediatric trauma. J Trauma 2001;50:1106-1110. DOI: 10.1097/00005373-200106000-00021
21. Prieto JM, Van Gen M, Calvo RY, et al. Nationwide analysis of resuscitative thoracotomy in pediatric trauma: time to differentiate from adult guidelines? J Trauma Acute Care Surg 2020;89(4):686-690. DOI: 10.1097/TA.0000000000002869. PMID: 33017132.

22. McLaughlin C, Zagory JA, Fenlon M, et al. Timing of mortality in pediatric trauma patients: A National Trauma Data Bank analysis. J Pediatr Surg 2018;53(2):344-351. DOI: 10.1016/j.jpedsurg.2017.10.006. Epub 2017. PMID: 29111081; PMCID: PMC5828917.

23. Finer NN, Peliowski A, Hayashi A. Tension hemopericardium complicating neonatal pneumopericardium. Am J Perinatol 1993;10(1):50-2. DOI: 10.1055/s-2007-994701 